\title{
Homogenized out-of-plane shear response of three-scale fiber-reinforced composites
}

\author{
Ariel Ramírez-Torres ${ }^{1} \cdot$ Raimondo Penta $^{2}$ - Reinaldo Rodríguez-Ramos ${ }^{4}$ - Alfio Grillo ${ }^{1}$. Luigi Preziosi ${ }^{1}$. \\ José Merodio ${ }^{3}$ - Raúl Guinovart-Díaz ${ }^{4}$. Julián Bravo-Castillero ${ }^{4}$
}

Received: 9 May 2017 / Accepted: 24 February 2018 / Published online: 29 June 2018

(c) The Author(s) 2018

\begin{abstract}
In the present work we embrace a three scales asymptotic homogenization approach to investigate the effective behavior of hierarchical linear elastic composites reinforced by cylindrical, uniaxially aligned fibers and possessing a periodic structure at each hierarchical level of organization. We present our novel results assuming isotropy of the constituents and focusing on the effective out-of-plane shear modulus, which is computed exploiting the solution of the arising anti-plane problems. The latter are solved semi-analytically by means of complex variables and successfully benchmarked against the results obtained by finite elements. Our findings can pave the way for multiscale modeling of complex hierarchical materials (such as bone and tendons) at a negligible computational cost.
\end{abstract}

Keywords Asymptotic homogenization $\cdot$ Hierarchical modeling $\cdot$ Linear elasticity $\cdot$ Multiscale composites $\cdot$ Biomimetic materials

Raimondo Penta

raimondo.penta@glasgow.ac.uk

Ariel Ramírez-Torres

ariel.ramirez@polito.it

Reinaldo Rodríguez-Ramos

reinaldo@matcom.uh.cu

Alfio Grillo

alfio.grillo@polito.it

Luigi Preziosi

luigi.preziosi@polito.it

José Merodio

jose.merodio@upm.es

Raúl Guinovart-Díaz

guino@matcom.uh.cu

Julián Bravo-Castillero

jbravo@matcom.uh.cu

1 Dipartimento di Scienze Matematiche "G. L. Lagrange", Politecnico di Torino, 10129 Turin, Italy

2 School of Mathematics and Statistics, Mathematics and Statistics Building, University of Glasgow, University Place, Glasgow G12 8QQ, UK

3 Departamento de Mecánica de los Medios Continuos y T. Estructuras, E.T.S. de Caminos, Canales y Puertos, Universidad Politécnica de Madrid, CP 28040 Madrid, Spain

\section{Introduction}

Multiscale composites organized across two or more length scales are often encountered in nature, as well as in artificial materials designed to optimize specific properties (see, e.g., [10]). Relevant applications involving hierarchical systems include, but are not limited to, rocks and fracture [11], biomechanics and nanomedicine [28], poroelasticity [21], and the bone tissue [26].

Multiscale modeling via suitable homogenization techniques is of crucial importance in the analysis of composite materials. On one hand, it can be exploited to obtain information concerning the coarser scale behavior of a system at hand (where experimental measurements are often easier to provide) on the basis of given microstructural properties of the finer scales. On the other hand, it can provide a deeper understanding on how to modify the microstructural arrangement of the finer scales constituents to achieve the optimal design of artificial constructs (e.g. biomimetic materials).

The most widely exploited techniques dealing with this issue in terms of mechanical properties rely on either the aver-

4 Departamento de Matemáticas, Facultad de Matemática y Computación, Universidad de La Habana, CP 10400 La Habana, Cuba 


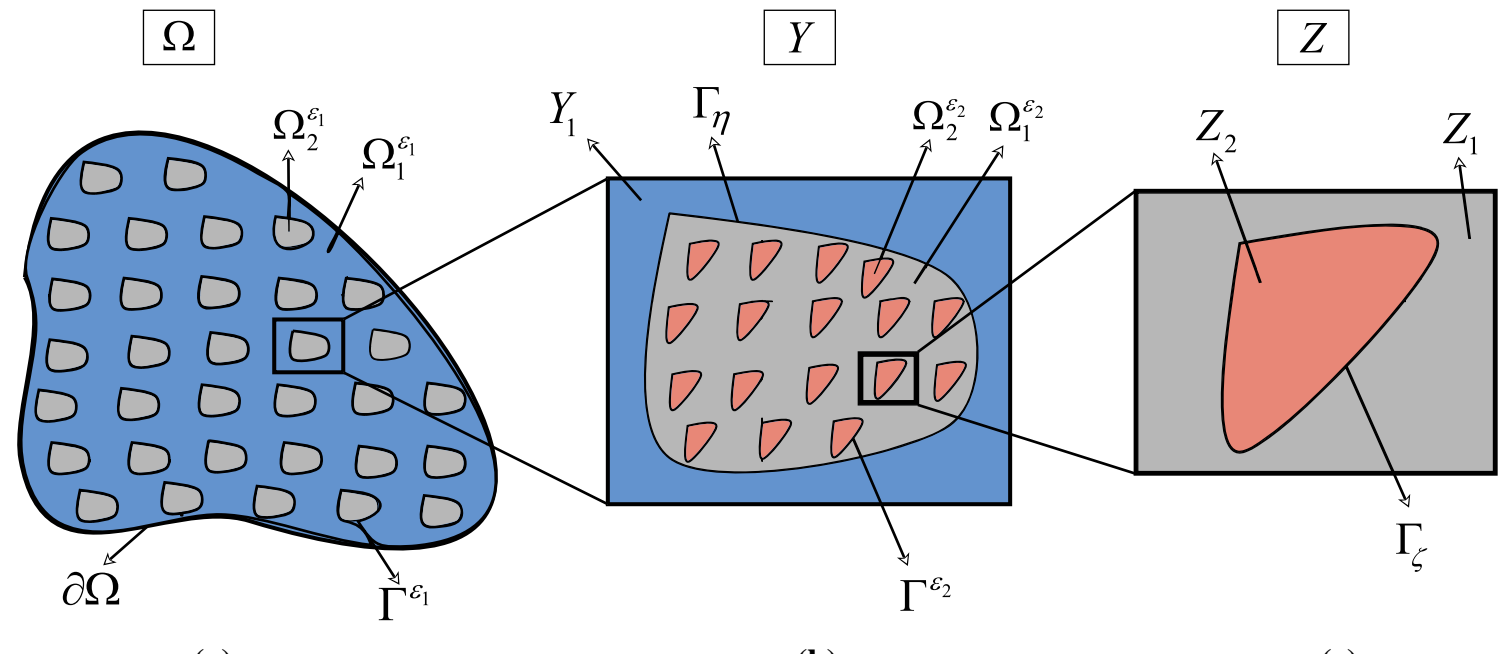

(a)

(b)

(c)

Fig. 1 Cross sections of the periodic composite: a whole material, $\mathbf{b} \varepsilon_{1}$-structural level. $\mathbf{c} \varepsilon_{2}$-structural level

age field techniques, such as Eshelby-based approaches or representative volume elements formulations, as well as the asymptotic homogenization technique, see, e.g., the review [9] for a comparison. The former have been much more extensively applied to complex, hierarchical systems, although also the computational potential and theoretical reliability of the asymptotic homogenization technique has been recently investigated (see, e.g., $[4,19,20]$ ). In particular, the reiterated homogenization technique $[1,3,12,30]$ has been considered in the theoretical study of heterogeneous composites presenting multiple length scales. From the application point of view, an extensively discussed hierarchical hard tissue has been bone and several hierarchical modeling via homogenization and continuum micromechanics methods have been used in the analysis of its mechanical properties [7,14,29].

In the present work we apply the three-scale asymptotic homogenization developed in [25] (which is therein applied to hierarchical layered materials) to investigate the effective behavior of fibrous hierarchical composites. The linear elastic constitutive framework and the homogenization steps developed in [25] are briefly illustrated in Sects. 2 and 3, respectively. We apply the latter to find the effective out-of-plane shear of a hierarchical linear elastic reinforced composite assuming a square-like arrangement of uniaxially aligned cylindrical fibers at all hierarchical levels of organization. We solve the resulting anti-plane cell problems (which are depicted in Sect. 4) for isotropic and piecewise constant materials properties using results of our previous works (see e.g. [5,6]). Our novel results (which match those computed numerically via finite elements) are presented and discussed in Sect. 5 in terms of the out-of-plane shear versus the fibers' volume fraction. We conclude the manuscript (cf. Sect. 6) highlighting possible applications to realistic scenarios of interest, such as hierarchical modeling of fibril bundles and/or fusing mineral crystals as analyzed for example in [22] in the context of aged bone modeling.

\section{The linear elastic problem}

Let us denote by $\Omega \subset \mathbb{R}^{3}$ a periodic composite possessing two hierarchical levels of organization (Fig. 1). The domain $\Omega$ at the first structural level (referred to as $\varepsilon_{1}$ ) is assumed to be reinforced by uniaxially aligned fibers, that is $\bar{\Omega}=$ $\bar{\Omega}_{1}^{\varepsilon_{1}} \cup \bar{\Omega}_{2}^{\varepsilon_{1}}$, where $\Omega_{2}^{\varepsilon_{1}}=\cup_{i=1}^{N} \Omega_{2}^{\varepsilon_{1}}$ denotes the ensemble of fibers and $\Omega_{1}^{\varepsilon_{1}}$ is the host medium at the $\varepsilon_{1}$ level. The interface between $\Omega_{1}^{\varepsilon_{1}}$ and $\Omega_{2}^{\varepsilon_{1}}$ is denoted by $\Gamma^{\varepsilon_{1}}$. At the second structural level (referred to as $\varepsilon_{2}$ ), each fiber ${ }_{i} \Omega_{2}^{\varepsilon_{1}}$ is supposed to be as well reinforced by aligned fibers oriented in the same direction of the composite fiber ${ }_{i} \Omega_{2}^{\varepsilon_{1}}$. Then, ${ }_{i} \Omega_{2}^{\varepsilon_{1}}=$ $\bar{\Omega}_{1}^{\varepsilon_{2}} \cup \bar{\Omega}_{2}^{\varepsilon_{2}}$, where $\Omega_{2}^{\varepsilon_{2}}=\cup_{j=1}^{M} \Omega_{2}^{\varepsilon_{2}}$ represents the ensemble of fibers and $\Omega_{1}^{\varepsilon_{2}}$ is the host medium at the $\varepsilon_{2}$-structural level. The interface between $\Omega_{1}^{\varepsilon_{2}}$ and $\Omega_{2}^{\varepsilon_{2}}$ is denoted by $\Gamma^{\varepsilon_{2}}$. At this stage, we assume that all constituents of the hierarchical composite behave as linear elastic materials with constitutive relationship for the stress tensor given by,

$\sigma=\mathbf{C}: \xi(\mathbf{u}), \quad$ with $\xi(\mathbf{u})=\frac{\nabla \mathbf{u}+\nabla \mathbf{u}^{T}}{2}$ in $\Omega$

where $\boldsymbol{\xi}(\mathbf{u})$ is the elastic strain tensor and $\mathbf{u}(\mathbf{x})$ is the elastic displacement at $\mathbf{x}=\left(x_{1}, x_{2}, x_{3}\right)$ with components $u_{1}(\mathbf{x}), u_{2}(\mathbf{x})$ and $u_{3}(\mathbf{x})$, being the components of $\mathbf{u}(\mathbf{x})$ in an orthonormal cartesian vector basis. The fourth rank tensor C with components $C_{i j k l}(i, j, k, l=1,2,3)$ is the stiffness tensor, which is assumed to be phase-wise smooth and positive definite and satisfies the standard symmetries, i.e., 
componentwise

$C_{i j k l}=C_{j i k l}=C_{i j l k}=C_{k l i j}, \quad C_{i j k l} \omega_{i j} \omega_{k l} \geq \varkappa \omega_{i j} \omega_{i j}$,

respectively, where $\omega$ is a second order symmetric tensor and $\varkappa>0$ is a constant. Then, ignoring inertia and volume forces, the problem in $\Omega$ reads

$\left(\mathbf{P}^{\varepsilon}\right) \begin{cases}\nabla \cdot\left[\mathbf{C}^{\varepsilon}: \boldsymbol{\xi}\left(\mathbf{u}^{\varepsilon}\right)\right]=0 & \text { in } \Omega \backslash\left(\Gamma^{\varepsilon_{1}} \cup \Gamma^{\varepsilon_{2}}\right) \\ \mathbf{u}^{\varepsilon}=\mathbf{u}^{*} & \text { on } \partial \Omega_{d}, \\ {\left[\mathbf{C}^{\varepsilon}: \boldsymbol{\xi}\left(\mathbf{u}^{\varepsilon}\right)\right] \cdot \mathbf{n}=\mathbf{S}^{*}} & \text { on } \partial \Omega_{n},\end{cases}$

where $\mathbf{n}$ is the outward unit vector normal to the surface $\partial \Omega$ and $\mathbf{u}^{*}$ and $\mathbf{S}^{*}$ are the prescribed displacement and traction on $\partial \Omega=\partial \Omega_{d} \cup \partial \Omega_{n}$ (with $\partial \Omega_{d} \cap \partial \Omega_{n}=\emptyset$ ), respectively. Moreover, continuity conditions for displacements and tractions are imposed on both $\Gamma^{\varepsilon_{1}}$ and $\Gamma^{\varepsilon_{2}}$, i.e.

$\llbracket \mathbf{u}^{\varepsilon} \rrbracket=0, \quad \llbracket \mathbf{C}^{\varepsilon}: \xi\left(\mathbf{u}^{\varepsilon}\right) \cdot \mathbf{n}_{\mathbf{j}} \rrbracket=0 \quad(\mathbf{j}=\boldsymbol{\eta}, \zeta)$,

where $\mathbf{n}_{\eta}=\left(n_{1}^{\eta}, n_{2}^{\eta}, n_{3}^{\eta}\right)$ and $\mathbf{n}_{\zeta}=\left(n_{1}^{\zeta}, n_{2}^{\zeta}, n_{3}^{\zeta}\right)$ represent the outward unit vectors to the surfaces $\Gamma^{\varepsilon_{1}}$ and $\Gamma^{\varepsilon_{2}}$, respectively. The operator $\llbracket \bullet \rrbracket$ denotes the jump across the interface between two constituents.

\section{Three scales asymptotic homogenization}

We consider three different scales, namely $d_{1}, d_{2}$ and $L$, which characterize the different structural sizes and we assume that they are well-separated, i.e.

$\varepsilon_{1}=\frac{d_{1}}{L} \ll 1$ and $\varepsilon_{2}=\frac{d_{2}}{d_{1}} \ll \varepsilon_{1}$.

Using relation (3), two formally independent variables

$\eta=\frac{\mathbf{x}}{\varepsilon_{1}} \quad$ and $\quad \zeta=\frac{\mathbf{x}}{\varepsilon_{2}}$

are introduced. Additionally, each field and material property is considered to be $\boldsymbol{\eta}$ and $\zeta$ periodic [15]. As a consequence of the performed spatial scale decoupling (4) and using the chain rule, it holds that

$\nabla \rightarrow \nabla_{\mathbf{x}}+\varepsilon_{1}^{-1} \nabla_{\eta}+\varepsilon_{2}^{-1} \nabla_{\zeta}$

where $\nabla_{\mathbf{j}}$ indicates that the derivative is performed with respect to $\mathbf{j}=\mathbf{x}, \boldsymbol{\eta}, \boldsymbol{\zeta}$. We now assume that the elastic displacement $\mathbf{u}^{\varepsilon}$ can be represented as a power series in terms of the small parameters $\varepsilon_{1}$ and $\varepsilon_{2}$, namely

$\mathbf{u}^{\varepsilon}(\mathbf{x}, \eta, \zeta)=\tilde{\mathbf{u}}^{0}(\mathbf{x}, \eta, \zeta)+\sum_{i=1}^{\infty} \tilde{\mathbf{u}}^{i}(\mathbf{x}, \eta, \zeta) \varepsilon_{2}^{i}$ where $\tilde{\mathbf{u}}^{(0)}$ is defined as

$\tilde{\mathbf{u}}^{0}(\mathbf{x}, \boldsymbol{\eta}, \zeta)=\mathbf{u}^{0}(\mathbf{x}, \boldsymbol{\eta}, \zeta)+\sum_{i=1}^{\infty} \mathbf{u}^{i}(\mathbf{x}, \boldsymbol{\eta}, \zeta) \varepsilon_{1}^{i}$.

Since the quantities involved vary on the $\eta$ and $\zeta$ scales, the following cell average operators are defined

$\langle\bullet\rangle_{\eta}=\frac{1}{|Y|} \int_{Y} \bullet \mathrm{d} \eta \quad$ and $\langle\bullet\rangle_{\zeta}=\frac{1}{|Z|} \int_{Z} \bullet \mathrm{d} \zeta$

where $|Y|$ and $|Z|$ represent the periodic cell volumes. Here and subsequently (unless necessary), the variable dependence is dropped out for convenience.

\subsection{Homogenization technique}

The homogenization technique, as depicted in [25], is sketched below. First, we substitute expansion (6) into (1) and (2), and then, use the chain rule and equate in powers of $\varepsilon_{2}$ "freezing" the small parameter $\varepsilon_{1}$. This allows to find the effective elastic properties at the $\varepsilon_{2}$-structural level and use the results as the inputs for the problems arising at the $\varepsilon_{1}$-structural level when equating in powers of $\varepsilon_{1}$. Finally, the effective coefficients of the problem are obtained. The procedure is detailed as follows:

Step 1 Substituting expansion (6) into (1); using result (5) and considering terms in powers of $\varepsilon_{2}$.

(i) To $O\left(\varepsilon_{2}^{0}\right)$

$\frac{\partial}{\partial \zeta_{j}}\left(C_{i j k l}^{\varepsilon} \xi_{k l}^{\zeta}\left(\tilde{\mathbf{u}}^{0}\right)\right)=0$ in $Z \backslash \Gamma_{\zeta}$

$\llbracket \tilde{u}_{i}^{0} \rrbracket=0$ on $\Gamma_{\zeta}$,

$\llbracket C_{i j k l}^{\varepsilon} \xi_{k l}^{\zeta}\left(\tilde{\mathbf{u}}^{0}\right) n_{j}^{\zeta} \rrbracket=0 \quad$ on $\Gamma_{\zeta}$

with

$\xi_{k l}^{h}(\mathbf{g})=\frac{1}{2}\left(\frac{\partial g_{k}}{\partial h_{l}}+\frac{\partial g_{l}}{\partial h_{k}}\right)$

where $\mathbf{g}$ is a vector. Since the right hand side of Eq. (8) is zero, the solvability condition is satisfied [2]. Then,

$\tilde{\mathbf{u}}^{0}=\tilde{\mathbf{u}}^{0}(\mathbf{x}, \boldsymbol{\eta}) \Leftrightarrow\left\{\begin{array}{l}\mathbf{u}^{0}=\mathbf{u}^{0}(\mathbf{x}, \boldsymbol{\eta}), \\ \mathbf{u}^{i}=\mathbf{u}^{i}(\mathbf{x}, \boldsymbol{\eta}),\end{array}\right.$

i.e. the homogeneity of (8) together with (9)-(10), leads to a periodic $\zeta$-constant solution. 
(ii) To $O\left(\varepsilon_{2}\right)$ and using the fact that $\xi_{k l}^{\zeta}\left(\tilde{\mathbf{u}}^{0}\right)=0$,

$$
\begin{aligned}
& \frac{\partial}{\partial \zeta_{j}}\left(C_{i j k l}^{\varepsilon} \xi_{k l}^{\zeta}\left(\tilde{\mathbf{u}}^{1}\right)\right) \\
& \quad=-\frac{\partial}{\partial \zeta_{j}}\left(C_{i j k l}^{\varepsilon}\left(\xi_{k l}^{x}\left(\tilde{\mathbf{u}}^{0}\right)+\varepsilon_{1}^{-1} \xi_{k l}^{\eta}\left(\tilde{\mathbf{u}}^{0}\right)\right)\right) \text { in } Z \backslash \Gamma_{\zeta}
\end{aligned}
$$

By the $\zeta$-periodicity of $\mathbf{C}^{\varepsilon}$ and the solvability condition, Eq. (11) has a $\zeta$-periodic solution which is unique up to an additive constant. In particular, since the problem is linear and $\tilde{\mathbf{u}}^{0}$ does not depend on $\zeta, \tilde{\mathbf{u}}^{1}$ can be written as

$\tilde{u}_{i}^{1}(\mathbf{x}, \boldsymbol{\eta}, \zeta)=\tilde{\chi}_{i k l}(\mathbf{x}, \boldsymbol{\eta}, \zeta) \tilde{U}_{k l}^{0}(\mathbf{x}, \boldsymbol{\eta})$

where

$\tilde{U}_{k l}^{0}=\xi_{k l}^{x}\left(\tilde{\mathbf{u}}^{0}(\mathbf{x}, \boldsymbol{\eta})\right)+\varepsilon_{1}^{-1} \xi_{k l}^{\eta}\left(\tilde{\mathbf{u}}^{0}(\mathbf{x}, \boldsymbol{\eta})\right)$.

The third rank tensor $\tilde{\chi}$ is $\boldsymbol{\eta}$ - and $\zeta$-periodic, and satisfies the local problem

$\frac{\partial}{\partial \zeta_{j}}\left(C_{i j k l}^{\varepsilon}+C_{i j p q}^{\varepsilon} \xi_{p q k l}^{\zeta}(\tilde{\chi})\right)=0$ in $Z \backslash \Gamma_{\zeta}$,

$\llbracket \tilde{\chi}_{i k l} \rrbracket=0$ on $\Gamma_{\zeta}$,

$\llbracket\left(C_{i j k l}^{\varepsilon}+C_{i j p q}^{\varepsilon} \xi_{p q k l}^{\zeta}(\tilde{\chi})\right) n_{j}^{\zeta} \rrbracket=0$ on $\Gamma_{\zeta}$

with

$\xi_{p q k l}^{h}(\mathbf{G})=\frac{1}{2}\left(\frac{\partial G_{p k l}}{\partial h_{q}}+\frac{\partial G_{q k l}}{\partial h_{p}}\right)$,

where $\mathbf{G}$ is a third rank tensor. Equations (13)-(15) constitute the $\varepsilon_{2}$-cell problem. The condition $\langle\tilde{\chi}\rangle_{\zeta}=0$ is imposed in order to guarantee uniqueness.

(iii) To $O\left(\varepsilon_{2}^{2}\right)$, applying the average operator $\langle\bullet\rangle_{\zeta}$ and taking into account the $\zeta$ periodicity of the involved functions,

$\left(\frac{\partial}{\partial x_{j}}+\varepsilon_{1}^{-1} \frac{\partial}{\partial \eta_{j}}\right) \check{C}_{i j k l}^{\varepsilon} \tilde{U}_{k l}^{0}=0$ in $\Omega_{1}^{\varepsilon_{1}}$,

where

$\check{C}_{i j k l}^{\varepsilon}=\left\langle C_{i j k l}^{\varepsilon}+C_{i j p q}^{\varepsilon} \xi_{p q k l}^{\zeta}(\tilde{\chi})\right\rangle_{\zeta}$

is the effective stiffness tensor at the $\varepsilon_{1}$-structural level. Note that the derivative in (16) depends on the small parameter $\varepsilon_{1}$ and $\check{\mathbf{C}}^{\varepsilon}=\check{\mathbf{C}}^{\varepsilon}(\mathbf{x}, \boldsymbol{\eta})$.
Step 2 Using (7) in (16) and equating in powers of $\varepsilon_{1}$.

(i) To $O\left(\varepsilon_{1}^{0}\right)$, we have

$\frac{\partial}{\partial \eta_{j}}\left(\check{C}_{i j k l}^{\varepsilon} \xi_{k l}^{\eta}\left(\mathbf{u}^{0}\right)\right)=0 \quad$ in $\quad Y \backslash \Gamma_{\eta}$

$\llbracket u_{i}^{0} \rrbracket=0$ on $\Gamma_{\eta}$,

$\llbracket \check{C}_{i j k l}^{\varepsilon} \xi_{k l}^{\eta}\left(\mathbf{u}^{0}\right) n_{j}^{\eta} \rrbracket=0$ on $\Gamma_{\eta}$.

Again the solvability condition is applied to (18)-(20), to obtain

$\mathbf{u}^{0}=\mathbf{u}^{0}(\mathbf{x})$.

(ii) To $O\left(\varepsilon_{1}\right)$, using the fact that $\xi_{k l}^{\eta}\left(\mathbf{u}^{0}\right)=0$ and applying the $\zeta$ average operator,

$\frac{\partial}{\partial \eta_{j}}\left(\check{C}_{i j k l}^{\varepsilon} \xi_{k l}^{\eta}\left(\mathbf{u}^{1}\right)\right)=-\frac{\partial}{\partial \eta_{j}}\left(\check{C}_{i j k l}^{\varepsilon} \xi_{k l}^{x}\left(\mathbf{u}^{0}\right)\right)$.

By the $\boldsymbol{\eta}$-periodicity of $\check{\mathbf{C}}^{\varepsilon}$ and the solvability condition, the above equation has a $\boldsymbol{\eta}$-periodic solution which is unique up to an additive constant. In particular, since the problem is linear and $\mathbf{u}^{0}$ does not depend on $\eta$,

$u_{i}^{1}(\mathbf{x}, \boldsymbol{\eta})=\chi_{i k l}(\mathbf{x}, \boldsymbol{\eta}) \xi_{k l}^{x}\left(\mathbf{u}^{0}(\mathbf{x})\right)$

where the third rank tensor $\chi$ is $\eta$-periodic and solution of

$\frac{\partial}{\partial \eta_{j}}\left(\check{C}_{i j k l}^{\varepsilon}+\check{C}_{i j p q}^{\varepsilon} \xi_{p q k l}^{\eta}(\chi)\right)=0$ in $Y \backslash \Gamma_{\eta}$,

$\llbracket \chi_{i k l} \rrbracket=0$ on $\Gamma_{\eta}$,

$\llbracket\left(\check{C}_{i j k l}^{\varepsilon}+\check{C}_{i j p q}^{\varepsilon} \xi_{p q k l}^{\eta}(\chi)\right) n_{j}^{\eta} \rrbracket=0$ on $\Gamma_{\eta}$.

Equations (22)-(24) represent the $\varepsilon_{1}$-cell problem. The condition $\langle\chi\rangle_{\eta}=0$ is imposed for guarantee uniqueness.

(iii) To $O\left(\varepsilon_{1}^{2}\right)$, applying the average operator $\langle\bullet\rangle_{\eta}$ and taking into account the $\boldsymbol{\eta}$ periodicity of the functions involved. The homogenized problem becomes

$\left(\mathbf{P}_{h}\right) \begin{cases}\frac{\partial}{\partial x_{j}}\left(\hat{C}_{i j k l} \xi_{k l}^{x}\left(\mathbf{u}^{0}\right)\right)=0 & \text { in } \Omega, \\ u_{i}^{0}=u_{i}^{*} & \text { on } \partial \Omega_{d}, \\ \hat{C}_{i j k l} \xi_{k l}^{x}\left(\mathbf{u}^{0}\right) n_{j}=S_{i}^{*} & \text { on } \partial \Omega_{n},\end{cases}$ 
where

$$
\hat{C}_{i j k l}=\left\langle\check{C}_{i j k l}^{\varepsilon}+\check{C}_{i j p q}^{\varepsilon} \xi_{p q k l}^{\eta}(\chi)\right\rangle_{\eta}
$$

is the effective stiffness tensor.

\section{Out-of-plane shear mechanical response}

Since we are considering uniaxially aligned fibers, the threedimensional cell problems can be equivalently formulated as two-dimensional problems over the cross-section of the cell. That is, by symmetry the auxiliary third rank tensors $\tilde{\chi}$ and $\chi$ do not depend on $\zeta_{3}$ and $\eta_{3}$, respectively. Therefore, we can refer to the spatial coordinates vectors as $\boldsymbol{\eta}=\left(\eta_{1}, \eta_{2}\right), \zeta=$ $\left(\zeta_{1}, \zeta_{2}\right)$ (keeping the same notation for the sake of simplicity), and the third components of the normal vectors $\mathbf{n}_{\zeta}$ and $\mathbf{n}_{\eta}$ reduce to zero, see also the appendix reported in [19].

We assume that $\mathbf{C}^{\varepsilon}$ is piecewise constant, such that, the parametric dependence of the $\varepsilon_{2}$-cell problem on the variables $\eta$ and $\mathbf{x}$ is lost and $\tilde{\chi}$ depends only on $\zeta$. As a consequence, $\check{\mathbf{C}}^{\varepsilon}$ is also piecewise constant (as it is averaged on $\zeta$ ), and therefore $\chi$ is depending only on $\boldsymbol{\eta}$, so that finally also $\hat{\mathbf{C}}$ is piecewise constant.

Applying the above mentioned dimensional reduction, and the fact that $\mathbf{C}^{\varepsilon}$ and $\check{\mathbf{C}}^{\varepsilon}$ are piecewise constants, the differential problems (13)-(15), (22)-(24) can be rewritten as

$$
\begin{cases}C_{i j p q}^{\gamma, \zeta} \frac{\partial^{2} \tilde{\chi}_{p k l}}{\partial \zeta_{j} \partial \zeta_{q}}=0 & \text { in } \tilde{Z}_{\gamma}, \\ \llbracket \tilde{\chi}_{i k l} \rrbracket=0 & \text { on } \tilde{\Gamma}_{\zeta}, \\ \llbracket\left(C_{i j k l}^{\zeta}+C_{i j p q}^{\zeta} \frac{\partial \tilde{\chi}_{p k l}}{\partial \zeta_{q}}\right) n_{j}^{\zeta} \rrbracket=0 & \text { on } \tilde{\Gamma}_{\zeta},\end{cases}
$$

and

$$
\begin{cases}C_{i j p q}^{\gamma, \eta} \frac{\partial^{2} \chi_{p k l}}{\partial \eta_{j} \partial \eta_{q}}=0 & \text { in } \tilde{Y}_{\gamma}, \\ \llbracket \chi_{i k l} \rrbracket=0 & \text { on } \tilde{\Gamma}_{\eta}, \\ \llbracket\left(C_{i j k l}^{\eta}+C_{i j p q}^{\eta} \frac{\partial \chi_{p k l}}{\partial \eta_{q}}\right) n_{j}^{\eta} \rrbracket=0 & \text { on } \tilde{\Gamma}_{\eta},\end{cases}
$$

respectively, with $j, q=1,2$ and $i, k, l, p=1,2,3$. In (26) and (27), $\tilde{Y}_{\gamma}$ and $\tilde{Z}_{\gamma}(\gamma=1,2)$, denote the two-dimensional unit cells at the $\varepsilon_{1}$ - and $\varepsilon_{2}$-structural levels, respectively, with restrictions to the matrix $(\gamma=1)$ and fiber $(\gamma=2)$. The interface curves between the constituents $\tilde{Y}_{1}, \tilde{Y}_{2}$ and $\tilde{Z}_{1}, \tilde{Z}_{2}$ are denoted by $\tilde{\Gamma}_{\eta}$ and $\tilde{\Gamma}_{\zeta}$, respectively. Moreover, $C_{i j k l}^{\gamma, \eta}$ and $C_{i j k l}^{\gamma, \zeta}$ are the stiffness tensors of the constituent $\gamma$ at the $\varepsilon_{1}$ and $\varepsilon_{2}$-structural levels, respectively. In particular, the stiffness tensor of the composite fiber at the $\varepsilon_{1}$ structural level is $C_{i j k l}^{2, \eta}=\check{C}_{i j k l}$.

We further assume that the constituents at the $\varepsilon_{2}$ level are isotropic, therefore the fiber phase at the $\varepsilon_{1}$-structural level can be at most monoclinic (i.e. 13 independent elastic coefficients), see, e.g. $[15,16]$. In our case, it is however tetragonal symmetric (6 independent elastic coefficients), as we are dealing with square-symmetric arrangement of cylindrical fibers (the cell's cross section corresponds to a square embedding a circle, see also [15,27]). In particular, the following relationships hold for both structural levels:

$C_{1313}^{\zeta}=C_{2323}^{\zeta}, \quad C_{1313}^{\eta}=C_{2323}^{\eta}$,

and, as both $\mathbf{C}^{\varepsilon}$ and $\check{\mathbf{C}}^{\varepsilon}$ are in particular orthotropic

$C_{1323}^{\zeta}=C_{2313}^{\zeta}=0, \quad C_{1323}^{\eta}=C_{2313}^{\eta}=0$

Here we focus on the shear mechanical response related to the third (out-of-plane) component of the elastic displacement. For a generic monoclinic material, the relevant constitutive relations are [15]

$\sigma_{13}=2 C_{1313} \xi_{13}+2 C_{1323} \xi_{23}$,

$\sigma_{23}=2 C_{2313} \xi_{13}+2 C_{2323} \xi_{23}$,

where $\sigma_{13}$ and $\sigma_{23}$ are the out-of-plane shear stresses and $\xi_{13}$ and $\xi_{23}$ are the shear strains. In our case, the resulting effective stiffness $\hat{\mathbf{C}}$ is still expected to be at most monoclinic (as the individual phases are tetragonal and the fibers are uniaxially aligned also on the $\varepsilon_{1}$-structural level).

We then aim at obtaining the effective elastic coefficients related to relationships (30) and (31), to characterize the outof-plane shear mechanical response. This can be done by performing a standard decomposition of the problems (26) and (27) into in-plane and antiplane problems. Exploiting (28) and (29), and fixing $i, p=3$ in (26) and (27), the resulting nontrivial anti-plane cell problems can be expressed in terms of the doubly periodic functions $\tilde{\chi}_{33 \alpha}^{\gamma}=\tilde{\chi}_{\alpha}^{\gamma}(\zeta)$ for $\zeta \in \tilde{Z}_{\gamma}$ and $\chi_{33 \alpha}^{\gamma}=\chi_{\alpha}^{\gamma}(\eta)$ for $\eta \in \tilde{Y}_{\gamma}$ as follows [15,27]

$\left(L_{\alpha}^{\zeta}\right) \begin{cases}\Delta_{\zeta}\left(\tilde{\chi}_{\alpha}^{\gamma}\right)=0 & \text { in } \tilde{Z}_{\gamma}, \\ \llbracket \tilde{\chi}_{\alpha} \rrbracket=0 & \text { on } \tilde{\Gamma}_{\zeta}, \\ \llbracket C_{1313}^{\zeta} \frac{\partial \tilde{\chi}_{\alpha}}{\partial \zeta_{j}} n_{j}^{\zeta} \rrbracket=-\llbracket C_{1313}^{\zeta} n_{\alpha}^{\zeta} \rrbracket & \text { on } \tilde{\Gamma}_{\zeta},\end{cases}$

and

$\left(L_{\alpha}^{\eta}\right) \begin{cases}\Delta_{\eta}\left(\chi_{\alpha}^{\gamma}\right)=0 & \text { in } \tilde{Y}_{\gamma}, \\ \llbracket \chi_{\alpha} \rrbracket=0 & \text { on } \tilde{\Gamma}_{\eta}, \\ \llbracket C_{1313}^{\eta} \frac{\partial \chi_{\alpha}}{\partial \eta_{j}} n_{j}^{\eta} \rrbracket=-\llbracket C_{1313}^{\eta} n_{\alpha}^{\eta} \rrbracket & \text { on } \tilde{\Gamma}_{\eta},\end{cases}$

with $\alpha=1,2$. Once solved the cell problem (32), the relevant effective coefficients at the $\varepsilon_{1}$ structural level are computed by 


$$
\begin{aligned}
& \check{C}_{\alpha 3 \alpha 3}=\left\langle C_{\alpha 3 \alpha 3}^{\zeta}+C_{\alpha 3 \alpha 3}^{\zeta} \frac{\partial \tilde{\chi}_{\alpha}}{\partial \zeta_{\alpha}}\right\rangle_{\zeta}, \\
& \check{C}_{1323}=\left\langle C_{2323}^{\zeta} \frac{\partial \tilde{\chi}_{1}}{\partial \zeta_{2}}\right\rangle_{\zeta}, \\
& \check{C}_{2313}=\left\langle C_{1313}^{\zeta} \frac{\partial \tilde{\chi}_{2}}{\partial \zeta_{1}}\right\rangle_{\zeta} .
\end{aligned}
$$

Solving (33), the effective coefficients are given by

$$
\begin{aligned}
& \hat{C}_{\alpha 3 \alpha 3}=\left\langle\check{C}_{\alpha 3 \alpha 3}+\check{C}_{\alpha 3 \alpha 3} \frac{\partial \chi_{\alpha}}{\partial \eta_{\alpha}}\right\rangle_{\eta}, \\
& \hat{C}_{1323}=\left\langle\check{C}_{2323} \frac{\partial \chi_{1}}{\partial \eta_{2}}\right\rangle_{\eta}, \\
& \hat{C}_{2313}=\left\langle\check{C}_{1313} \frac{\partial \chi_{2}}{\partial \eta_{1}}\right\rangle_{\eta} .
\end{aligned}
$$

Since we assume square-symmetry also on the $\varepsilon_{1}$ structural level, the resulting effective stiffness tensor is actually expected to exhibit tetragonal symmetry, so that in particular

$\hat{C}_{1313}=\hat{C}_{2323} ; \quad \hat{C}_{1323}=\hat{C}_{2313}=0$,

as also shown by the results that follow. The effective shear mechanical response is then completely characterized by the effective out-of-plane shear modulus $\hat{C}_{1313}$.

\section{Results and discussion}

The theory of analytical functions in [13] can be applied to solve the cell problems (32) and (33), where doubly periodic harmonic functions need to be found (See "Appendix A"). In order to compute the effective properties at both structural levels, it is necessary to truncate the systems (46) and (47) into appropriate orders. Then,

$$
\begin{aligned}
& \check{C}_{1313}-\mathrm{i} \check{C}_{2313}=C_{1313}^{1, \zeta}\left(1-2 \pi \overline{\tilde{a}}_{1}\right), \\
& \check{C}_{1323}-\mathrm{i} \check{C}_{2323}=-C_{1313}^{1, \zeta}\left(1+2 \pi \overline{\tilde{a}}_{1}\right) \mathrm{i}, \\
& \hat{C}_{1313}-\mathrm{i} \hat{C}_{2313}=C_{1313}^{1, \eta}\left(1-2 \pi \bar{a}_{1}\right), \\
& \hat{C}_{1323}-\mathrm{i} \hat{C}_{2323}=-C_{1313}^{1, \eta}\left(1+2 \pi \bar{a}_{1}\right) \mathrm{i},
\end{aligned}
$$

where $\bar{a}_{1}\left(\overline{\tilde{a}}_{1}\right)$ denotes the conjugate of the complex coefficient $a_{1}\left(\tilde{a}_{1}\right)$ and " $\mathrm{i}$ " is the imaginary unit.

Using formulas (41)-(44) the effective coefficients at both structural levels can be found. For the sake of exemplifying, we choose the following material properties $C_{1313}^{1, \zeta}=1$, $C_{1313}^{2, \zeta}=10$ and $C_{1313}^{1, \eta}=5$, and made a parametric study by varying the volume fraction of the fiber at both structural levels. Particularly, the effective coefficients are computed for the same fiber volume fraction at each structural level, i.e. $\phi=\phi_{\zeta}=\phi_{\eta}$. Then, we proceed in the following way:

1. Given the properties $C_{1313}^{1, \zeta}$ and $C_{1313}^{2, \zeta}$ and for a fixed volume fraction of $\tilde{Z}_{2}$, denoted by $\phi_{\zeta}$, the cell problem at the $\varepsilon_{2}$-structural level (32) is solved. To find the coefficient $\tilde{a}_{l}$, the infinite linear system (46) is truncated at a certain order $\tilde{K}$ and solved. The matrix $\mathbf{W}_{k}^{\zeta}$ is written in terms of $\phi_{\zeta}$.

2. To compute the effective coefficients $\check{C}_{1313}, \check{C}_{1323}, \check{C}_{2313}$ and $\check{C}_{2323}$ of the composite fiber $\Omega_{2}^{\varepsilon_{1}}$, the solution $\tilde{a}_{l}$ of the linear system is substituted in (41) and (42).

3. Now, the cell problem at the $\varepsilon_{1}$-structural level (33) is solved. In particular, we only need to fix $C_{1313}^{1, \eta}$ since $C_{1313}^{2, \eta}=\check{C}_{1313}$ was found in the previous step. At this point the volume fraction of the fiber $\tilde{Y}_{2}$ must be fixed. One way to proceed is to make $\phi_{\eta}=\phi_{\zeta}$.

4. Finally, to find $a_{l}$, and consequently the effective coefficients $\hat{C}_{1313}, \hat{C}_{1323}, \hat{C}_{2313}$ and $\hat{C}_{2323}$, the infinite linear system (47) is truncated at a certain order $K$ and solved. In this case, the matrix $\mathbf{W}_{k}^{\eta}$ is equal to $\mathbf{W}_{k}^{\zeta}$, which is already computed.

Figure 2 presents the values obtained for the effective coefficients $\check{C}_{1313}$ (left) and $\hat{C}_{1313}$ (right) as a function of the fiber volume fraction. Specifically, the truncation order of the systems was fixed to $\tilde{K}=K=3$, thus, decreasing the computational cost. As a consequence of constituent's isotropic behavior, we obtain $\check{C}_{1313}=\check{C}_{2323}, \hat{C}_{1313}=\hat{C}_{2323}$ and $\breve{C}_{2313}=\check{C}_{1323}=\hat{C}_{2313}=\hat{C}_{1323}=0$. A comparison between the results by the three scales asymptotic homogenization method and the finite element method using FreeFEM++ is also shown in Fig. 2. Specifically, we approximate the involved functions by piecewise linear continuous finite elements. As observed, the curves overlap even when the truncation orders of the infinite linear systems are very small. Interestingly, for the particular set of parameters fixed, the effective behavior of $\hat{C}_{1313}$ (in the context of elasticity $\hat{C}_{1313}$ is the effective shear modulus $\hat{\mu}$ ) behaves as a quadratic curve in function of the fiber volume fraction. This behavior is a consequence of the fact that at the $\varepsilon_{2}$-structural level the fibers are stiffer than the host medium, and at the $\varepsilon_{1}$-structural level the matrix becomes the stiffer constituent. In several works similar patterns have been observed. For instance, the enhancement of the effective out-of-plane Young modulus was obtained in [24] for a periodic bilaminate, and in [31], for a particular type of composite material.

\section{Concluding remarks}

In the present work, the effective behavior of hierarchical linear elastic composites at different length scales was studied. 

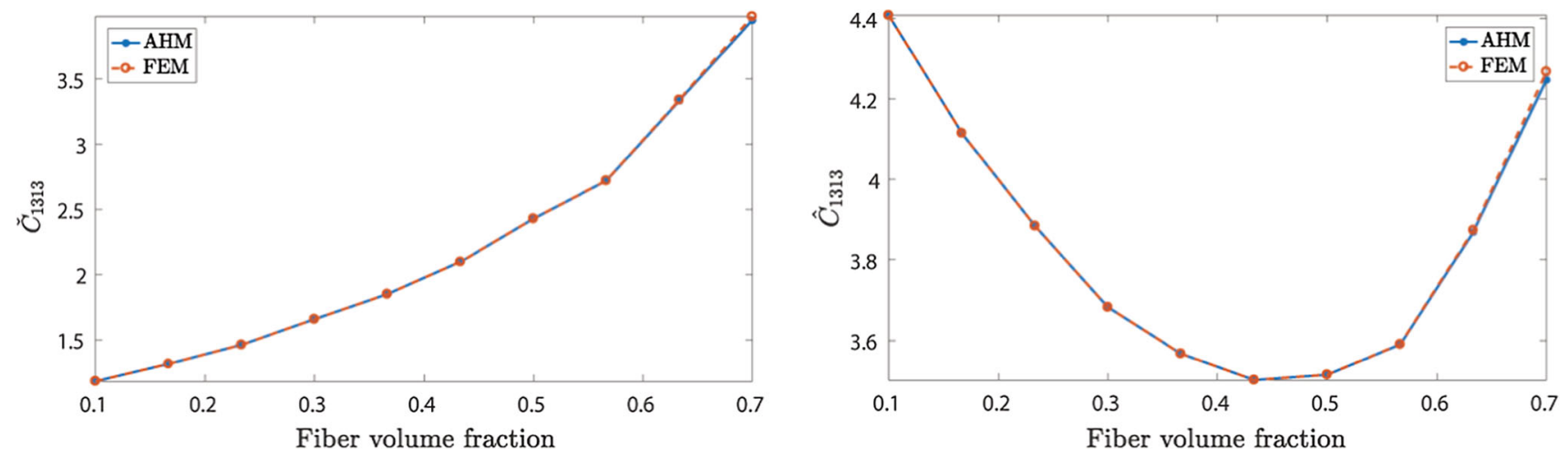

Fig. 2 Effective out-of-plane shear moduli $\check{C}_{1313}$ (formula (41)) (left) and $\hat{C}_{1313}$ (formula (43)) (right) plotted against the fiber volume fraction. Comparisons with FEM are also shown

The approach permits the study of problems where several length scales are present (the two-scale asymptotic homogenization only deals with two different length scales). The novelty of this work relies in the solution, for the first time, of the effective elastic shear stiffness considering a nested arrangement of cylindrical and uniaxially aligned fibers. The corresponding cell problems are solved analytically using complex variables and the results are compared with a finite element approach. Since analytical formulas are found for computing the effective coefficients the computational cost is very low.

Future developments of this work will concern the analysis of the in-plane problems $[16,27]$ to fully characterize the effective mechanical response of hierarchical threescale materials. Moreover, the extension of the present model to interface debonding conditions (see, e.g. [23]), as well as geometric heterogeneities (for example generalizing the non-macroscopically uniform homogenization approach developed in $[8,17,18]$ ) will provide more general and realistic results applicable to real-world hierarchical composites. The current method represents a first step towards computationally feasible multiscale modeling of complex hierarchical materials. For example, this approach could be exploited to model musculoskeletal mineralized tissues (such as bone and tendons) which are organized across several spatial scales. In this context, the three scales would represent the arragement on the basic constituents (i.e. collagen and mineral crystals), the resulting mineralized collagen fibril, and finally their packing into the mineralized collagen fibril bundle. Since the model is developed for fiber-reinforced composites, it can then serve as an approximation for both fibers embedded in a matrix, and for fusing inclusions of reinforcing material, such as for example the hydroxyapatite mineral crystals which are found in the aged bone tissue (see, e.g. [22]).

Acknowledgements AR (“titolare di un Assegno di Ricerca dell' Istituto Nazionale di Alta Matematica"), AG and LP are financially supported by Indam. JM and RP acknowledge support from the Ministery of Econ- omy in Spain under the project reference DPI2014-58885-R. RR, RG and JB, thank the Project (7515) "Métodos Físico-Matemáticos para el estudio de nuevos materiales y la propagación de ondas. Aplicaciones". AG is funded by the "Politecnico di Torino" and the "Fondazione Cassa di Risparmio di Torino" in the context of the funding campaign "La Ricerca dei Talenti" (HR Excellence in Research). JB recognizes the support of Cátedra IIMAS-UNAM.

Open Access This article is distributed under the terms of the Creative Commons Attribution 4.0 International License (http://creativecomm ons.org/licenses/by/4.0/), which permits unrestricted use, distribution, and reproduction in any medium, provided you give appropriate credit to the original author(s) and the source, provide a link to the Creative Commons license, and indicate if changes were made.

\section{Appendix A: Analytic solution of the cell problems}

The doubly periodic harmonic functions are sought in terms of the following expansions of harmonic functions [13]:

$\tilde{\chi}_{k}^{\gamma}=\operatorname{Re}\left\{\tilde{\varphi}^{\gamma}(z)\right\} \quad$ and $\quad \chi_{k}^{\gamma}=\mathcal{R} e\left\{\varphi^{\gamma}(z)\right\}$,

where

$\tilde{\varphi}^{1}=\sum_{l=1}^{\infty o}\left(\tilde{a}_{l} z^{-l}-\sum_{m=1}^{\infty o} m \Lambda_{m l} \tilde{a}_{m} z^{l}\right), \quad \tilde{\varphi}^{2}=\sum_{l=1}^{\infty o} \tilde{c}_{l} z^{l}$, $\varphi^{1}=\sum_{l=1}^{\infty o}\left(a_{l} z^{-l}-\sum_{m=1}^{\infty o} m \Lambda_{m l} a_{m} z^{l}\right), \quad \varphi^{2}=\sum_{l=1}^{\infty o} c_{l} z^{l}$,

with $\tilde{a}_{l}, a_{l}, \tilde{c}_{l}$ and $c_{l}$ being complex coefficients to be determined, $\Lambda_{11}=(-1)^{k+1} \pi$ and $\Lambda_{m l}=S_{m+l}(m+l-1) ! / m ! l !$. The reticulate sums are defined by $S_{m}=\sum_{w \in L^{*}} w^{-m}$ ( $m>3, m$ odd) for $w=r w_{1}+s w_{2}$ with $r, s \in \mathbb{Z}$ and $L^{*}$ represents the lattice excluding $w=0$. The superscript " $o$ " indicates that the sum is carried out only over odd indices.

After substituting (45) in the local problems (32) and (33) and some algebraic manipulations. The following systems of 
equations are obtained in order to find the complex coefficients $\tilde{a}_{m}$ and $a_{m}$,

$$
\begin{aligned}
& \left(\begin{array}{l}
\tilde{A}_{1} \\
\tilde{A}_{2}
\end{array}\right)=\left[\left(\begin{array}{ll}
\mathbf{I} & 0 \\
\mathbf{0} & \mathbf{I}
\end{array}\right)+(-1)^{k+1} \Upsilon \zeta\left(\begin{array}{cc}
{ }_{k} \mathbf{W}_{1}^{\zeta} & -{ }_{k} \mathbf{W}_{2}^{\zeta} \\
-{ }_{k} \mathbf{W}_{2}^{\zeta} & -{ }_{k} \mathbf{W}_{1}^{\zeta}
\end{array}\right)\right]^{-1} \mathbf{V}_{k}^{\zeta}, \\
& \left(\begin{array}{l}
A_{1} \\
A_{2}
\end{array}\right)=\left[\left(\begin{array}{ll}
\mathbf{I} & 0 \\
\mathbf{0} & \mathbf{I}
\end{array}\right)+(-1)^{k+1} \Upsilon \eta\left(\begin{array}{cc}
{ }_{k} \mathbf{W}_{1}^{\eta} & -{ }_{k} \mathbf{W}_{2}^{\eta} \\
-{ }_{k} \mathbf{W}_{2}^{\eta} & -{ }_{k} \mathbf{W}_{1}^{\eta}
\end{array}\right)\right]^{-1} \mathbf{V}_{k}^{\eta},
\end{aligned}
$$

where $\mathbf{I}$ is the infinite identity matrix, $\mathbf{0}$ is the infinite zero matrix, $\tilde{A}_{1}=\left({ }_{1} \tilde{a}_{1},{ }_{1} \tilde{a}_{3}, \ldots\right), \tilde{A}_{2}=\left({ }_{2} \tilde{a}_{1},{ }_{2} \tilde{a}_{3}, \ldots\right)$, $\tilde{a}_{l}={ }_{1} \tilde{a}_{l}+{ }_{2} \tilde{a}_{l} I\left(I^{2}=-1\right), A_{1}=\left({ }_{1} a_{1},{ }_{1} a_{3}, \ldots\right), A_{2}=$ $\left({ }_{2} a_{1},{ }_{2} a_{3}, \ldots\right), a_{l}={ }_{1} a_{l}+{ }_{2} a_{l} \mathbf{i},{ }_{k} \mathbf{W}^{\mathbf{j}}={ }_{k} \mathbf{W}_{1}^{\mathbf{j}}+{ }_{k} \mathbf{W}_{2}{ }^{\mathbf{j}}$ $(\mathbf{j}=\zeta, \eta)$ with

${ }_{k} \mathbf{W}^{\mathbf{j}}= \begin{cases}(-1)^{k+1} \pi R_{\mathbf{j}}^{2} & \text { for } m+l=2, \\ \sum_{m=1}^{\infty o} \sqrt{m l} \Lambda_{m l} R_{\mathbf{j}}^{m+l} & \text { for } m+l>2,\end{cases}$

where $R_{\zeta}$ and $R_{\eta}$ represent the fiber radius of $\tilde{Z}_{2}$ and $\tilde{Y}_{2}$, respectively. Besides, $\mathbf{V}_{k}^{\zeta}=\left((-1)^{k+1} \Upsilon^{\zeta} R_{\zeta}, 0, \ldots\right), \mathbf{V}_{k}^{\eta}=$ $\left((-1)^{k+1} \Upsilon^{\eta} R_{\eta}, 0, \ldots\right)$ and

$\Upsilon^{\zeta}=\frac{C_{1313}^{1, \zeta}-C_{1313}^{2, \zeta}}{C_{1313}^{1, \zeta}+C_{1313}^{2, \zeta}}$ and $\Upsilon^{\eta}=\frac{C_{1313}^{1, \eta}-C_{1313}^{2, \eta}}{C_{1313}^{1, \eta}+C_{1313}^{2, \eta}}$

\section{References}

1. Allaire, G., Briane, M.: Multiscale convergence and reiterated homogenisation. Proc. R. Soc. Edinb. Sect. A Math. 126(02), 297342 (1996)

2. Bakhvalov, N.S., Panasenko, G.: Homogenisation: Averaging Processes in Periodic Media: Mathematical Problems in the Mechanics of Composite Materials, vol. 36. Springer, Dordrecht (1989)

3. Bensoussan, A., Lions, J.L., Papanicolaou, G.: Asymptotic Analysis for Periodic Structures, vol. 5. North-Holland Publishing Company, Amsterdam (1978)

4. Espinosa-Almeyda, Y., Camacho-Montes, H., Rodríguez-Ramos, R., Guinovart-Díaz, R., López-Realpozo, J.C., Bravo-Castillero, J., Sabina, F.J.: Influence of imperfect interface and fiber distribution on the antiplane effective magneto-electro-elastic properties for fiber reinforced composites. Int. J. Solids Struct. 112, 155-168 (2017)

5. Guinovart-Díaz, R., López-Realpozo, J., Rodríguez-Ramos, R., Bravo-Castillero, J., Ramírez, M., Camacho-Montes, H., Sabina, F.: Influence of parallelogram cells in the axial behaviour of fibrous composite. Int. J. Eng. Sci. 49, 75-84 (2011)

6. Guinovart-Díaz, R., Yan, P., Rodríguez-Ramos, R., LópezRealpozo, J., Jiang, C., Bravo-Castillero, J., Sabina, F.: Effective properties of piezoelectric composites with parallelogram periodic cells. Int. J. Eng. Sci. 53, 58-66 (2012)

7. Hamed, E., Lee, Y., Jasiuk, I.: Multiscale modeling of elastic properties of cortical bone. Acta Mech. 213(1), 131-154 (2010)
8. Holmes, M.: Introduction to Perturbation Method. Springer, New York (1995)

9. Hori, M., Nemat-Nasser, S.: On two micromechanics theories for determining micromacro relations in heterogeneous solids. Mech. Mater. 31, 667-682 (1999)

10. Kim, C.S., Randow, C., Sano, T., et al.: Hybrid and Hierarchical Composite Materials. Springer, Switzerland (2015)

11. Lee, S.H., Lough, M., Jensen, C.: Hierarchical modeling of flow in naturally fractured formations with multiple length scales. Water Resour. Res. 37(3), 443-455 (2001)

12. Lukkassen, D., Milton, G.W.: On hierarchical structures and reiterated homogenization. In: Function Spaces, Interpolation Theory and Related Topics (Lund, 2000), pp. 355-368 (2002)

13. Muskhelishvili, N.I.: Some Basic Problems of the Mathematical Theory of Elasticity. Springer, Berlin (1977)

14. Nikolov, S., Raabe, D.: Hierarchical modeling of the elastic properties of bone at submicron scales: the role of extrafibrillar mineralization. Biophys. J. 94(11), 4220-4232 (2008)

15. Parnell, W.J., Abrahams, I.D.: Dynamic homogenization in periodic fibre reinforced media. Quasi-static limit for SH waves. Wave Motion 43(6), 474-498 (2006)

16. Parnell, W.J., Abrahams, I.D.: Homogenization for wave propagation in periodic fibre-reinforced media with complex microstructure. I. Theory. J. Mech. Phys. Solids 56(7), 2521-2540 (2008)

17. Penta, R., Ambrosi, D., Quarteroni, A.: Multiscale homogenization for fluid and drug transport in vascularized malignant tissues. Math. Models Methods Appl. Sci. 25(1), 79-108 (2015)

18. Penta, R., Ambrosi, D., Shipley, R.J.: Effective governing equations for poroelastic growing media. Q. J. Mech. Appl. Math. 67(1), 6991 (2014)

19. Penta, R., Gerisch, A.: Investigation of the potential of asymptotic homogenization for elastic composites via a three-dimensional computational study. Comput. Vis. Sci. 17(4), 185-201 (2015)

20. Penta, R., Gerisch, A.: The asymptotic homogenization elasticity tensor properties for composites with material discontinuities. Contin. Mech. Thermodyn. 29, 187-206 (2017)

21. Penta, R., Merodio, J.: Homogenized modeling for vascularized poroelastic materials. Meccanica 52(14), 3321-3343 (2017)

22. Penta, R., Raum, K., Grimal, Q., Schrof, S., Gerisch, A.: Can a continuous mineral foam explain the stiffening of aged bone tissue? A micromechanical approach to mineral fusion in musculoskeletal tissues. Bioinspiration Biomim. 11(3), 035004 (2016)

23. Qu, J.: The effect of slightly weakened interfaces on the overall elastic properties of composite materials. Mech. Mater. 14(4), 269281 (1993)

24. Ramírez, M., Nava-Gómez, G.G., Sabina, F.J., Camacho-Montes, H., Guinovart-Díaz, R., Rodríguez-Ramos, R., Bravo-Castillero, J.: Enhancement of youngs moduli and auxetic windows in laminates with isotropic constituents. Int. J. Eng. Sci. 58, 95-114 (2012)

25. Ramírez-Torres, A., Penta, R., Rodríguez-Ramos, R., Merodio, J., Sabina, F.J., Bravo-Castillero, J., Guinovart-Díaz, R., Preziosi, L., Grillo, A.: Three scales asymptotic homogenization and its application to layered hierarchical hard tissues. Int. J. Solids Struct. 130, 190-198 (2018)

26. Rho, J.Y., Kuhn-Spearing, L., Zioupos, P.: Mechanical properties and the hierarchical structure of bone. Med. Eng. Phys. 20(2), 92102 (1998)

27. Rodríguez-Ramos, R., Sabina, F.J., Guinovart-Díaz, R., BravoCastillero, J.: Closed-form expressions for the effective coefficients of a fiber-reinforced composite with transversely isotropic constituents-i. Elastic and square symmetry. Mech. Mater. 33, 223-235 (2001)

28. Taffetani, M., de Falco, C., Penta, R., Ambrosi, D., Ciarletta, P.: Biomechanical modelling in nanomedicine: multiscale approaches and future challenges. Arch. Appl. Mech. 84(9-11), 1627-1645 (2014) 
29. Telega, J.J., Gałka, A., Tokarzewski, S.: Application of the reiterated homogenization to determination of the effective moduli of compact bone. J. Theor. Appl. Mech. 37, 687-706 (1999)

30. Trucu, D., Chaplain, M., Marciniak-Czochra, A.: Three-scale convergence for processes in heterogeneous media. Appl. Anal. 91(7), 1351-1373 (2012)
31. Zhu, H.X., Fan, T.X., Zhang, D.: Composite materials with enhanced dimensionless young's modulus and desired poissons ratio. Sci. Rep. 5, 14103 (2015). https://doi.org/10.1038/srep14103 